\title{
COMBINATORICS FOR GENERAL KINETICALLY CONSTRAINED SPIN MODELS
}

\author{
LAURE MARÊCHÉ
}

\begin{abstract}
We study the set of possible configurations for a general kinetically constrained model $(\mathrm{KCM})$, a non monotone version of the $\mathcal{U}$-bootstrap percolation cellular automata. We solve a combinatorial question that is a generalization of a problem addressed by Chung, Diaconis and Graham in 2001 for a specific one-dimensional KCM, the East model. Since the general models we consider are in any dimension and lack the oriented character of the East dynamics, we have to follow a completely different route than the one taken by Chung, Diaconis and Graham. Our combinatorial result is used by Marêché, Martinelli and Toninelli to complete the proof of a conjecture put forward by Morris.
\end{abstract}

2010 Mathematics subject classification: Primary 60K35, Secondary 05C75.

Key words. Kinetically constrained models, bootstrap percolation.

\section{INTRODUCTION}

In this article, we study a generalization of a combinatorial problem addressed by Chung, Diaconis and Graham in' [8], that can be formulated as follows. Fix $N \in \mathbb{N}$ and consider that any element of $\{-N, \ldots, N\}$ (we call them sites) can be in state 0 or 1 . The configuration of states can change with respect to the following rules: there eannot be two state changes at the same time, and the state of a site can change only if its left neighbor is in state zero. We consider that the sites outside $\{-N, \ldots, N\}$ have state 0 . One of the questions tackled in [8] is: if the initial configuration contains only ones in $\{-N, \ldots, N\}$ and if there can only be $n$ zeroes in $\{-N, \ldots, N\}$ at the \$ame time, is it possible to place a zero at the origin with these rules ? Chung, Diaconis and Graham proved that it is possible if and only if $N \leq 2^{n}-2$ : the bigger $N$ is, the bigger $n$ has to be (a non rigorous version of this proof was given previously by Sollich and Evans in [15]).

This problem was motivated by the study of the East model [9], a stochastic particle system defined as follows: ach site of $\mathbb{Z}$ can be in state 0 or 1 , and is updated (independently) at rate one by setting it to 0 with probability and to 1 with probability $1-q$, if and only if its left neighbor is at zero. Indeed, the above combinatorial result is one of the key ingredients to determine the relevant time scales for the East dynamics [1, 6]. The East model belongs to a more general class of interacting particle systems, called kinetically constrained models (KCM), that were introduced by physicists to model the liquid/glass transition, an important open problem of condensed matter pohysics (see for example [14, 3] for reviews). In order to construct a different KCM, we use the same dynamics as for East, but with a different choice of the constraint that has to be satisfied to update a site. For example, if one allows a site to change state when its left or its right neighbor is at 0 (this is the choice corresponding to the जso-called Fredrickson-Andersen one spin facilitated model (FA1f)), the behavior is entirely different: for any value of $N$, two zeroes at the same time are always enough to reach the origin. Indeed, we can put the site $-N$ at 0 , then put $-N+1$ at 0 , then put $-N$ at 1 , put $-N+2$ at 0 , put $-N+1$ at 1 , etc. and we end up reaching the origin, using never more than two zeroes at the same time.

In this article, we study a generalization of the combinatorial problem of Chung, Diaconis and Graham in higher dimension and with totally general rules. Though our motivation comes from the study of KCM, we stress that the content of this paper is purely deterministic and requires no probabilistic tools. Let us give a precise definition of

I acknowledge support of the ERC Starting Grant 680275 MALIG. 
the class of rules that we address. We set $d \in \mathbb{N}^{*}, N \in \mathbb{N}$; any site of $\{-N, \ldots, N\}^{d}$ can be in state 0 or 1 . There cannot be two state changes at the same time, and the state of a site $s$ can change only if there exists $X \in \mathcal{U}$ such that all the sites of $s+X$ are in state 0 , where $\mathcal{U}=\left\{X_{1}, \ldots, X_{m}\right\}$ with $m \in \mathbb{N}^{*}$ and the $X_{i}$ are finite nonempty subsets of $\mathbb{Z}^{d} \backslash\{0\}\left(\mathcal{U}\right.$ is called an update family and the $X_{i}$ are called update rules). As before, the sites outside $\{-N, \ldots, N\}^{d}$ are considered to be in state 0 . The rules of the East model correspond to $d=1$ and $\mathcal{U}=\{\{-1\}\}$, and those of the FA1f model to $d=1$ and $\mathcal{U}=\{\{-1\},\{1\}\}$. If the initial configuration contains only ones in $\{-N, \ldots, N\}^{d}$ and if there can only be $n$ zeroes in $\{-N, \ldots, N\}^{d}$ at the same time, is it possible to place a zero at the origin?

This generalization has become interesting in recent years. Indeed, until a few years ago, only specific update families had been studied in KCM. However, there recently was a breakthrough in the study of a monotone deterministic counterpart of KCM called bootstrap percolation. For any update family $\mathcal{U}$ of $\mathbb{Z}^{d}$, the associated bootstrap percolation process is defined as follows: we choose a set $A \subset \mathbb{Z}^{d}$ of sites that we consider as intially infected (the equivalent of being at zero), we set $A_{0}=A$, and for any $t \in \mathbb{N}^{*}$ we define the set $A_{t}$ of sites that are infected at time $t$ by

$$
A_{t}=A_{t-1} \cup\left\{s \in \mathbb{Z}^{d} \mid \exists X \in \mathcal{U}, s+X \subset A_{t-1}\right\},
$$

which means that at each time $t \in \mathbb{N}^{*}$, the sites that were infected at time $t-1$ remain infected at time $t$ and a site $s$ that was not infected at time $t-1$ becomes infected at time $t$ if and only if there exists $X \in \mathcal{U}$ such that all the sites of $s+X$ are infected at time $t-1$.

The articles [5] by Bollobás, Smith and Uzzell and [2] by Balister, Bollobás, Przykucki, and Smith tackled general update families for the first time and proved a beautiful universality result. They showed that in $\mathbb{Z}^{2}$, the update families can be sorted into three classes (whose definitions are too technical to be given in this introduction): subcritical, critical and supercritical, which have different behaviors that we are going to describe. The first natural question for a bootstrap percolation model is: if we start the process with each site having probability $q$ to be infected, independently of the others, will the process infect the origin with probability 1 or is there a positive probability that the origin is never infected even if we wait for an infinite time? Moreover, what will be the scale of the first time at which the origin is infected (often called infection time) ? Since bootstrap percolation is monotonic (the more infection we have at the beginning, the more we will have at any stage), it can be seen that there exists a critical probability $q_{c} \in[0,1]$ such that if $q<q_{c}$, the origin is never infected with positive probability and if $q>q_{c}$ the origin is infected with probability 1. [5, 2] showed that when $\mathcal{U}$ is subcritical, $q_{c}>0$, and when $\mathcal{U}$ is critical or supercritical, $q_{c}=0$. Moreover, they proved that when $q$ tends to zero, the infection time scales as $1 / q^{\Theta(1)}$ when $\mathcal{U}$ is supercritical and as $\exp \left(1 / q^{\Theta(1)}\right)$ when $\mathcal{U}$ is critical (the latter result was later refined by Bollobás, Duminil-Copin, Morris, and Smith in [4]).

These results call for the study of KCM with general update families. As in bootstrap percolation, a key quantity for the study of KCM is the first time at which the origin is at zero when the process starts with all sites independently at zero with probability $q$; we denote its mean by $\tau(q)$. Understanding the divergence of $\tau(q)$ when $q$ tends to $q_{c}$ is particularly relevant, because the critical regime $q \downarrow q_{c}$ is the most interesting for physicists. An easy result proven by Martinelli and Toninelli in [12] shows that the infection time in the bootstrap percolation process is a lower bound for $\tau(q)$. However, this lower bound does not always give the actual behavior. Indeed, for the East model, the infection time in the bootstrap percolation scales as $1 / q^{\Theta(1)}$ when $q$ tends to 0 , but the results of Aldous and Diaconis [1] and Cancrini, Martinelli, Roberto and Toninelli [6] proved that $\tau(q)$ scales as $\exp \left(\Theta\left(\log (1 / q)^{2}\right)\right)$ when $q$ tends to 0 . This lead Morris to formulate conjectures on the scaling of $\tau(q)$ when $q$ tends to zero for critical and supercritical update families. His conjecture for supercritical update families (conjecture 2.7 of [13]) is that they should be divided in two subclasses: supercritical unrooted update families for which $\tau(q)$ has the same scaling as the bootstrap percolation infection time, that is $1 / q^{\Theta(1)}$, and supercritical rooted update families for which $\tau(q)$ has the same scaling as the East model, $\exp \left(\Theta\left(\log (1 / q)^{2}\right)\right)$. Part of this conjecture was 
proven: the lower bound for supercritical unrooted update families is given by the bootstrap percolation lower bound of [12, and the upper bound for supercritical update families both unrooted and rooted was proven by Martinelli, Morris and Toninelli in [11. However, the lower bound for supercritical rooted update families was still missing. Since a lower bound matching this behavior for the East model was proven in [7] using the combinatorial result of [8], we seeked to generalize this combinatorial result to all supercritical rooted update families.

Indeed, we establish the following result (theorem 4 ): if $\mathcal{U}$ is a supercritical rooted update family, if we start with all the sites of $\{-N, \ldots, N\}^{2}$ at state 1 and if we allow only $n$ zeroes at the same time in $\{-N, \ldots, N\}^{2}$, then to be able to put a zero at the origin, it is necessary to have $N=O\left(n 2^{n}\right)$. This result is almost optimal, since [8] proved that for the East model, which is supercritical rooted, $N=2^{n}-2$ allows to put a zero at the origin. Actually, our result is valid in an even larger class, namely for all update families that are not supercritical unrooted. Furthermore, in proposition 6 we also explain why our hypothesis is not restrictive, namely why such a result is not valid for supercritical unrooted update families. Our result allows us to complete the proof of the conjecture of Morris (with respect to $\tau(q)$ ), which we do in theorem 4.2 of [10] with Martinelli and Toninelli. Our result proves even more, since it is valid in any dimension for a natural generalization of the definition of supercritical unrooted update families.

Though we generalize the result of [8], our proof is completely different from theirs, as the proof of [8] relies heavily on the orientation of the East model and the general update families completely lack orientation. Note that even in dimension 1, it is a substantial generalization of the result of [8], because it applies to a whole class of update families instead of just the East model.

We begin this article by giving the notations and stating the results, then we detail the proof of the result for one-dimensional supercritical rooted update families, then we explain how this proof extends to general dimension, and finally we examine the supercritical unrooted case.

\section{Notations AND RESUlt}

We fix $d \in \mathbb{N}^{*}$ and set an update family $\mathcal{U}=\left\{X_{1}, \ldots, X_{m}\right\}$ with the $X_{i}$ finite nonempty subsets of $\mathbb{Z}^{d} \backslash\{0\}$. Set $\Lambda \subset \mathbb{Z}^{d}$. We consider the configurations of states in $\Lambda$; they belong to the set $\{0,1\}^{\Lambda}$. We denote by $1_{\Lambda}$ the configuration which contains only ones in $\Lambda$, and by $0_{\Lambda}$ (or just 0 ) the configuration which contains only zeroes in $\Lambda$. Furthermore, for all $\eta \in\{0,1\}^{\Lambda}, s \in \Lambda$, we use the notation $\eta^{s}$ for the configuration in $\{0,1\}^{\Lambda}$ that is $\eta$ apart from the state of $s$ that is flipped: $\left(\eta^{s}\right)_{s^{\prime}}=1-\eta_{s}$ if $s^{\prime}=s$ and $\eta_{s^{\prime}}$ if $s^{\prime} \neq s$. Moreover, if $\Lambda^{\prime} \subset \Lambda$ and $\eta \in\{0,1\}^{\Lambda}$, we denote by $\eta_{\Lambda^{\prime}}$ its restriction to $\Lambda^{\prime}$. In addition, if $\Lambda^{\prime} \subset \mathbb{Z}^{d}$ is disjoint from $\Lambda$, for all $\eta \in\{0,1\}^{\Lambda}, \eta^{\prime} \in\{0,1\}^{\Lambda^{\prime}}$, we denote by $\eta_{\Lambda} \eta_{\Lambda^{\prime}}^{\prime}$ the configuration on $\Lambda \cup \Lambda^{\prime}$ defined by $\left(\eta_{\Lambda} \eta_{\Lambda^{\prime}}^{\prime}\right)_{s}=\eta_{s}$ if $s \in \Lambda$ and $\left(\eta_{\Lambda} \eta_{\Lambda^{\prime}}^{\prime}\right)_{s}=\eta_{s}^{\prime}$ if $s \in \Lambda^{\prime}$. We say that a move from $\eta \in\{0,1\}^{\Lambda}$ to $\eta^{\prime} \in\{0,1\}^{\Lambda}$ is legal if $\eta^{\prime}=\eta$, or if $\eta^{\prime}=\eta^{s}$ with $s \in \Lambda$ and there exists an update rule $X \in \mathcal{U}$ such that $\left(\eta_{\Lambda} 0_{\Lambda^{c}}\right)_{s+X}=0_{s+X}$ (we may also write $\left(\eta_{\Lambda}\right)_{s+X}=0$ to simplify the notation); that is, a move is legal if it respects the rules described in the introduction, assuming that all sites outside of $\Lambda$ are zeroes.

Definition 1. If $\eta, \eta^{\prime} \in\{0,1\}^{\Lambda}$, a legal path from $\eta$ to $\eta^{\prime}$ is a sequence of configurations $\left(\eta^{j}\right)_{0 \leq j \leq m}$ such that $m \in \mathbb{N}^{*}, \eta^{0}=\eta, \eta^{m}=\eta^{\prime}$, and for all $j \in\{0, \ldots, m-1\}$, the move from $\eta^{j}$ to $\eta^{j+1}$ is legal.

For any $n \in \mathbb{N}$, we say that $\left(\eta^{j}\right)_{0 \leq j \leq m}$ is an $n$-legal path if for all $j \in\{0, \ldots, m\}, \eta^{j}$ does not contain more than $n$ zeroes in $\Lambda$.

In order to have lighter notation, we use the same notation $\eta^{j}$ for the $j$-th step of a path and for the configuration that is equal to $\eta$ everywhere except at site $j$. In order to avoid confusion, $\eta^{0}, \eta^{j}, \eta^{j+1}$ and $\eta^{m}$ will always denote a step of a path, and no other index will be used to describe a step of a path.

For all $n \in \mathbb{N}$, we define

$$
V(n, \Lambda)=\left\{\eta \in\{0,1\}^{\Lambda} \mid \text { there exists an } n \text {-legal path from } 1_{\Lambda} \text { to } \eta\right\} .
$$


$V(n, \Lambda)$ is the set of configurations of $\{0,1\}^{\Lambda}$ that are attainable from the configuration containing only ones using at most $n$ zeroes. $V(n, \Lambda)$ will be very different depending on the properties of $\mathcal{U}$. In this article, we will distinguish between two classes of update families. To define them, we recall the concept of stable direction introduced in [5]:

Definition 2. For any $u \in S^{d-1}$, let $\mathbb{H}_{u}=\left\{x \in \mathbb{R}^{d} \mid\langle x, u\rangle<0\right\}$ the half-space with boundary orthogonal to $u$. We say that $u$ is a stable direction for the update family $\mathcal{U}$ when there does not exist $X \in \mathcal{U}$ such that $X \subset \mathbb{H}_{u}$.

This implies in particular that if we apply the rules in $\mathbb{Z}^{d}$ with the update family $\mathcal{U}$, and if we start with only ones in $\left(\mathbb{H}_{u}\right)^{c}$, then no zero can appear in $\left(\mathbb{H}_{u}\right)^{c}$. Intuitively, it means that the zeroes cannot move towards direction $u$. The following definition is an extension to the dimension $d$ of the definition proposed in [13]:

Definition 3. We say that $\mathcal{U}$ is supercritical unrooted if there exists a hyperplane of $\mathbb{R}^{d}$ that contains all stable directions of $\mathcal{U}$.

An example of supercritical unrooted update family is the one corresponding to the Fredrickson-Andersen one spin facilitated model, whose one-dimensional version was presented in the introduction, for which $\mathcal{U}=$ $\left\{\left\{e_{1}\right\}, \ldots,\left\{e_{d}\right\},\left\{-e_{1}\right\}, \ldots,\left\{-e_{d}\right\}\right\}$ where $\left\{e_{1}, \ldots, e_{d}\right\}$ is the canonical basis of $\mathbb{R}^{d}$. This update family has no stable directions at all.

We are now ready to state our main result, theorem 4, which is valid for all update families that are not supercritical unrooted. This actually covers many different behaviors; in particular, in two dimensions, according to the classification in [5] they include: supercritical update families which have two non opposite stable directions (called supercritical rooted in [13]), critical and subcritical update families.

Theorem 4. Let $\mathcal{U}$ be any update family that is not supercritical unrooted. There exists a constant $\kappa>0$ such that for any $n \in \mathbb{N}$, every $\eta \in V\left(n,\left\{-\left\lfloor\kappa n 2^{n}\right\rfloor, \ldots,\left\lfloor\kappa n 2^{n}\right\rfloor\right\}^{d}\right)$ satisfies $\eta_{0}=1$.

Remark 5. Our theorem is stated for paths that are $n$-legal when all sites outside of the box $\left\{-\left\lfloor\kappa n 2^{n}\right\rfloor, \ldots,\left\lfloor\kappa n 2^{n}\right\rfloor\right\}^{d}$ are considered to be zeroes; it actually remains valid if we consider the $n$-legal paths for any configuration of the states outside of the box. Indeed, if we consider that the sites outside of the box are not all zeroes, the possible moves are more restricted, hence a legal path for such a configuration is also a legal path if there are zeroes outside of the box.

The assumption that $\mathcal{U}$ is not supercritical unrooted in theorem 4 is not restrictive. Indeed, if $\mathcal{U}$ is supercritical unrooted, the behavior is different:

Proposition 6. If $d=1$ or 2 , and if $\mathcal{U}$ is supercritical unrooted, there exists $n \in \mathbb{N}^{*}$ such that for any domain $\Lambda \subset \mathbb{Z}^{d}$ containing the origin, there exists $\eta \in V(n, \Lambda)$ such that $\eta_{0}=0$.

Proposition 6 means that there exists a finite $n$ such that $n$ zeroes are always enough to bring a zero to the origin. We expect this result to hold also for $d \geq 3$. A sketch of proof can be found in section 5.

\section{The one-Dimensional CASE}

Let $\mathcal{U}$ be a one-dimensional, non supercritical unrooted update family. Then $\mathcal{U}$ has at least one stable direction, which can be 1 or -1 . Without loss of generality, we may suppose that -1 is a stable direction. We denote $r$ the range of the interactions: $r=\max \left\{\|x-y\|_{\infty} \mid x, y \in X \cup\{0\}, X \in \mathcal{U}\right\}$. Moreover, for all $n \in \mathbb{N}$, we write $a_{n}=r\left(2^{n}-1\right), b_{n}=r n 2^{n-1}$ and $\mathcal{P}_{n}=\left\{-a_{n}, \ldots, b_{n}\right\}$.

We will prove theorem 4 by induction. For all $n \in \mathbb{N}$, we denote

$$
\mathcal{H}_{n}=\text { "for any } \Lambda \subset \mathbb{Z} \text { such that } \mathcal{P}_{n} \subset \Lambda \text {, for any } \eta \in V(n, \Lambda), \eta_{0}=1 \text { ". }
$$

Proving $\mathcal{H}_{n}$ for all $n \in \mathbb{N}$ will prove the theorem in the one-dimensional case.

In order to do that, we will need the 


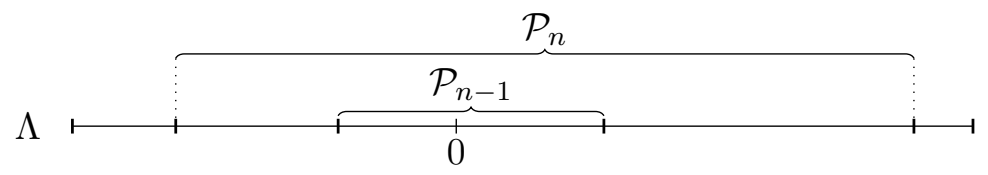

Figure 1. Proof of the theorem in the one-dimensional case: there must be a zero in $\Lambda \backslash \mathcal{P}_{n-1}$, hence there can be at most $n-1$ zeroes in $\mathcal{P}_{n-1}$. Thus $\mathcal{H}_{n-1}$ implies that there is no zero at 0 .

Lemma 7. Let $n \geq 1$ and suppose $\mathcal{H}_{n-1}$. Then, for all $\Lambda \subset \mathbb{Z}$ such that $\mathcal{P}_{n} \subset \Lambda$, for all $\eta \in V(n, \Lambda) \backslash\left\{1_{\Lambda}\right\}$, $\eta$ has at least one zero in $\Lambda \backslash \mathcal{P}_{n-1}$.

This lemma means that if $\mathcal{H}_{n-1}$ holds, in a large enough interval, any configuration attainable using no more than $n$ zeroes must have one of its zeroes outside of $\mathcal{P}_{n-1}$ (except the configuration containing only ones, that has no zero at all). This implies that there are at most $n-1$ zeroes in $\mathcal{P}_{n-1}$, which will allow us to use $\mathcal{H}_{n-1}$ to prove that the origin cannot be reached by zeroes (see figure 1).

We first prove the theorem supposing lemma 7 holds; we will prove the lemma afterwards. As we announced, we will show by induction that $\mathcal{H}_{n}$ holds for any $n \in \mathbb{N}$.

Case $n=0$. This is a simple case: if $\Lambda \subset \mathbb{Z}, \mathcal{P}_{0} \subset \Lambda$ and $\eta \in V(0, \Lambda)$, then $\eta$ contains no zero.

Induction. Let $n \geq 1$. We suppose $\mathcal{H}_{n-1}$. Let us show $\mathcal{H}_{n}$. Let $\Lambda \subset \mathbb{Z}$ such that $\mathcal{P}_{n} \subset \Lambda$, and $\eta \in V(n, \Lambda)$.

By definition, there exists an $n$-legal path $\left(\eta^{j}\right)_{0 \leq j \leq m}$ from $1_{\Lambda}$ to $\eta$. We will prove that $\left(\eta_{\mathcal{P}_{n-1}}^{j}\right)_{0 \leq j \leq m}$ is an $(n-1)$-legal path from $\eta_{\mathcal{P}_{n-1}}^{0}=1_{\mathcal{P}_{n-1}}$ to $\eta_{\mathcal{P}_{n-1}}^{m}=\eta_{\mathcal{P}_{n-1}}$.

Firstly, for all $j \in\{0, \ldots, m-1\}$, the move from $\eta_{\mathcal{P}_{n-1}}^{j}$ to $\eta_{\mathcal{P}_{n-1}}^{j+1}$ is legal. Indeed, if $\eta^{j+1}=\eta^{j}$ or if $\eta^{j+1}=\left(\eta^{j}\right)^{z}$ with $z \in \Lambda \backslash \mathcal{P}_{n-1}, \eta_{\mathcal{P}_{n-1}}^{j+1}=\eta_{\mathcal{P}_{n-1}}^{j}$ and the move from $\eta_{\mathcal{P}_{n-1}}^{j+1}$ to $\eta_{\mathcal{P}_{n-1}}^{j}$ is legal. Furthermore, if $\eta^{j+1}=\left(\eta^{j}\right)^{z}$ with $z \in \mathcal{P}_{n-1}, \eta_{\mathcal{P}_{n-1}}^{j+1}=\left(\eta_{\mathcal{P}_{n-1}}^{j}\right)^{z}$, and since the move from $\eta^{j}$ to $\eta^{j+1}$ is legal, there exists $X \in \mathcal{U}$ such that $\left(\eta_{\Lambda}^{j} 0_{\Lambda^{c}}\right)_{z+X}=0$, which implies $\left(\eta_{\mathcal{P}_{n-1}}^{j} 0_{\left(\mathcal{P}_{n-1}\right)^{c}}\right)_{z+X}=0$, hence the move from $\eta_{\mathcal{P}_{n-1}}^{j}$ to $\eta_{\mathcal{P}_{n-1}}^{j+1}$ is legal. Therefore $\left(\eta_{\mathcal{P}_{n-1}}^{j}\right)_{0 \leq j \leq m}$ is a legal path.

Moreover, for all $j \in\{0, \ldots, m\}, \eta_{\mathcal{P}_{n-1}}^{j}$ contains at most $n-1$ zeroes. Indeed, if $\eta^{j}=1_{\Lambda}$, then $\eta_{\mathcal{P}_{n-1}}^{j}$ contains no zero at all. In addition, if $\eta^{j} \neq 1_{\Lambda}$, then $\eta^{j} \in V(n, \Lambda) \backslash\left\{1_{\Lambda}\right\}$, and since we suppose $\mathcal{H}_{n-1}$, we can apply lemma 7. which yields that $\eta^{j}$ has at least one zero in $\Lambda \backslash \mathcal{P}_{n-1}$, hence $\eta_{\mathcal{P}_{n-1}}^{j}$ contains at most $n-1$ zeroes.

It follows that $\left(\eta_{\mathcal{P}_{n-1}}^{j}\right)_{0 \leq j \leq m}$ is an $(n-1)$-legal path from $1_{\mathcal{P}_{n-1}}$ to $\eta_{\mathcal{P}_{n-1}}$. Thus $\eta_{\mathcal{P}_{n-1}} \in V\left(n-1, \mathcal{P}_{n-1}\right)$. Consequently, by $\mathcal{H}_{n-1}, \eta_{0}=1$, which proves $\mathcal{H}_{n}$.

This ends the proof of theorem 4 given lemma 7 , so we are only left to prove lemma 7 .

Proof of lemma 7 , Let $n \geq 1$ and $\Lambda \subset \mathbb{Z}$ be such that $\mathcal{P}_{n} \subset \Lambda$.

We will consider a configuration $\eta \in\{0,1\}^{\Lambda}$, different from $1_{\Lambda}$, containing at most $n$ zeroes, such that all of its zeroes are in $\mathcal{P}_{n-1}$, and we will show that $\eta \notin V(n, \Lambda)$; this is enough to prove the lemma.

We begin by noticing that if there does not exist an $n$-legal path from $\eta$ to $1_{\Lambda}$, then $\eta \notin V(n, \Lambda)$. Indeed, if $\eta \in V(n, \Lambda)$, there exists an $n$-legal path $\left(\eta^{j}\right)_{0 \leq j \leq m}$ from $1_{\Lambda}$ to $\eta$, and one can check that $\left(\eta^{m-j}\right)_{0 \leq j \leq m}$ is an $n$-legal path from $\eta$ to $1_{\Lambda}$. Therefore, to prove that $\eta \notin V(n, \Lambda)$, it is enough to show that there is no $n$-legal path from $\eta$ to $1_{\Lambda}$. In order to do that, we let $\left(\eta^{j}\right)_{0 \leq j \leq m}$ be an $n$-legal path with $\eta^{0}=\eta$. We are going to show that $\eta^{m}$ cannot be $1_{\Lambda}$. 
$\Lambda$

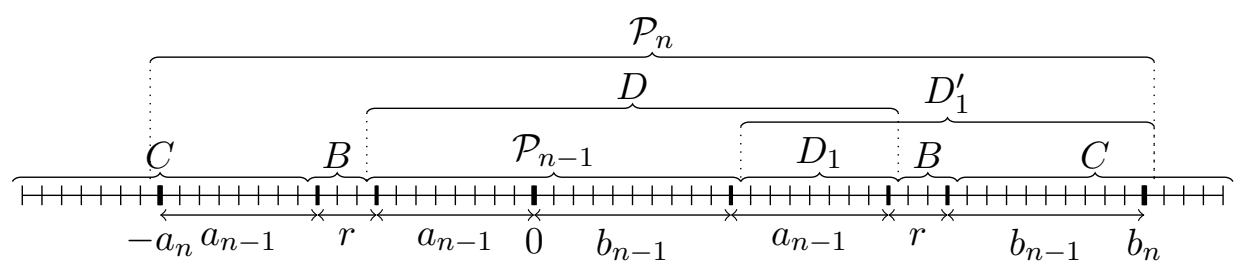

Figure 2. The setting of lemma 7

To this end, we will denote (see figure 2):

$$
\begin{aligned}
B & =\left\{-a_{n}+a_{n-1}, \ldots,-a_{n}+a_{n-1}+r-1\right\} \cup\left\{b_{n}-\left(b_{n-1}+r\right)+1, \ldots, b_{n}-b_{n-1}\right\}, \\
D & =\left\{-a_{n}+a_{n-1}+r, \ldots, b_{n}-\left(b_{n-1}+r\right)\right\}, \\
D_{1} & =\left\{b_{n}-\left(b_{n-1}+a_{n-1}+r\right)+1, \ldots, b_{n}-\left(b_{n-1}+r\right)\right\}, \\
D_{1}^{\prime} & =\left\{b_{n}-\left(b_{n-1}+a_{n-1}+r\right)+1, \ldots, b_{n}\right\}
\end{aligned}
$$

and $C=\Lambda \backslash(B \cup D)$ (if $n=1, D_{1}$ will be empty).

We notice that

$$
-a_{n}+a_{n-1}+r=-r\left(2^{n}-1\right)+r\left(2^{n-1}-1\right)+r=-r 2^{n-1}+r=-r\left(2^{n-1}-1\right)=-a_{n-1}
$$

and

$$
b_{n}-\left(b_{n-1}+a_{n-1}+r\right)=r n 2^{n-1}-\left(r(n-1) 2^{n-2}+r\left(2^{n-1}-1\right)+r\right)=r n 2^{n-2}-r 2^{n-2}=r(n-1) 2^{n-2}=b_{n-1}
$$

hence $\mathcal{P}_{n-1}=\left\{-a_{n}+a_{n-1}+r, \ldots, b_{n}-\left(b_{n-1}+a_{n-1}+r\right)\right\}=D \backslash D_{1}$.

$B$ will be a "buffer zone": we will prove that it remains full of ones and prevents the zeroes of $C$ and $D$ from interacting.

There will always be a zero in $\mathcal{P}_{n-1}$, because the leftmost zero $z$ in $\mathcal{P}_{n-1}$ would need an update rule full of zeroes to disappear. However, there is no zero in $B$ and the thickness of $B$ is larger than the range of the interactions, hence this update rule cannot use zeroes in $B$ or at the left of $B$. Thus it can use only zeroes in $\mathcal{P}_{n-1}$ or at the right of $\mathcal{P}_{n-1}$, but $z$ is the leftmost zero in $\mathcal{P}_{n-1}$. Therefore, the update rule would have to be completely contained in the right of $z$, which is impossible since we assumed that -1 was a stable direction, hence there is no update rule contained in $\mathbb{N}^{*}$. Hence the leftmost zero in $\mathcal{P}_{n-1}$ cannot disappear, thus there will always be a zero in $\mathcal{P}_{n-1}$, which implies $\eta^{m} \neq 1_{\Lambda}$.

More rigorously, we are going to prove by induction on $j \in\{0, \ldots, m\}$ that the property $\mathcal{H}_{j}^{\prime}$ holds, where $\mathcal{H}_{j}^{\prime}$ consists in:

$\left(P_{1}^{j}\right) \eta_{\mathcal{P}_{n-1}}^{j}$ contains a zero.

$\left(P_{2}^{j}\right) \eta_{B}^{j}=1_{B}$.

$\left(P_{3}^{j}\right) \eta_{C}^{j} 1_{\Lambda \backslash C} \in V(n-1, \Lambda)$.

$\left(P_{4}^{j}\right) \eta_{D_{1}}^{j} 1_{D_{1}^{\prime} \backslash D_{1}} \in V\left(n-1, D_{1}^{\prime}\right)$.

The last two properties will be used to show that $B$ remains full of ones.

If we can show $\mathcal{H}_{j}^{\prime}$ for all $j \in\{0, \ldots, m\}$, in particular $\left(P_{1}^{m}\right)$ will imply that there is a zero in $\eta_{\mathcal{P}_{n-1}}^{m}$, thus $\eta^{m} \neq 1_{\Lambda}$, which is enough to prove the lemma.

Let us prove $\mathcal{H}_{j}^{\prime}$ for all $j \in\{0, \ldots, m\}$. 
Case $j=0$.

$\left(P_{1}^{0}\right)$ is true, because $\eta^{0}=\eta \neq 1_{\Lambda}$, so $\eta$ contains at least a zero, and by assumption all zeroes of $\eta$ are in $\mathcal{P}_{n-1}$. $\left(P_{2}^{0}\right)$ is true because $\eta^{0}=\eta$ has no zero in $\Lambda \backslash \mathcal{P}_{n-1}$. $\left(P_{3}^{0}\right)$ is true because $C \subset \Lambda \backslash \mathcal{P}_{n-1}$, thus $\eta_{C}=1_{C}$, hence $\eta_{C}^{0} 1_{\Lambda \backslash C}=1_{\Lambda} \in V(n-1, \Lambda) . \quad\left(P_{4}^{0}\right)$ is true, because $D_{1} \subset \Lambda \backslash \mathcal{P}_{n-1}$, thus $\eta_{D_{1}}^{0} 1_{D_{1}^{\prime} \backslash D_{1}}=1_{D_{1}^{\prime}} \in V\left(n-1, D_{1}^{\prime}\right)$. Consequently, $\mathcal{H}_{0}^{\prime}$ holds.

Induction.

Let $j$ be in $\{0, \ldots, m-1\}$. We suppose that $\mathcal{H}_{j}^{\prime}$ holds. Let us show $\mathcal{H}_{j+1}^{\prime}$.

We know that the move from $\eta^{j}$ to $\eta^{j+1}$ is legal. If $\eta^{j+1}=\eta^{j}, \mathcal{H}_{j+1}^{\prime}$ holds because $\mathcal{H}_{j}^{\prime}$ holds. In the following, we deal with the case $\eta^{j+1}=\left(\eta^{j}\right)^{z}$ where $z \in \Lambda$ and there exists $X \in \mathcal{U}$ with $\left(\eta_{\Lambda}^{j}\right)_{z+X}=0$. The arguments will depend on the position of $z$.

Case $z \in B$.

We will show that $z \in B$ is impossible: the buffer zone remains preserved at step $j+1$.

By $\left(P_{2}^{j}\right) \eta_{B}^{j}=1_{B}$, hence $z+X \subset C \cup \Lambda^{c} \cup D$. Moreover, if there existed $x \in(z+X) \cap\left(C \cup \Lambda^{c}\right)$ and $y \in(z+X) \cap D$, then we would get $|x-y|>r$, which is impossible by the definition of $r$. Therefore $z+X \subset C \cup \Lambda^{c}$ or $z+X \subset D$. We are going to deal with the two cases separately.

We begin with the case $z+X \subset C \cup \Lambda^{c}$.

We are going to prove that in this case, $\left(\eta_{C}^{j} 1_{\Lambda \backslash C}\right)^{z}$ would be in $V(n-1, \Lambda)$, which is impossible because it has a zero at $z$ and $z+\mathcal{P}_{n-1} \subset \Lambda$, therefore $\mathcal{H}_{n-1}$ and the invariance by translation of $\mathbb{Z}$ yield a contradiction. Indeed, the move from $\eta_{C}^{j} 1_{\Lambda \backslash C}$ to $\left(\eta_{C}^{j} 1_{\Lambda \backslash C}\right)^{z}$ would be legal. In addition, $\left(\eta_{C}^{j} 1_{\Lambda \backslash C}\right)^{z}$ would coincide with $\eta^{j+1}$ on $C \cup B$ by $\left(P_{2}^{j}\right)$. Moreover, $\eta^{j+1}$ contains at most $n$ zeroes, and $\eta_{\mathcal{P}_{n-1}}^{j+1}=\eta_{\mathcal{P}_{n-1}}^{j}$ would contain at least a zero by $\left(P_{1}^{j}\right)$, hence $\eta^{j+1}$ contains at most $n-1$ zeroes in $C \cup B$, thus $\left(\eta_{C}^{j} 1_{\Lambda \backslash C}\right)^{z}$ would contain at most $n-1$ zeroes. Furthermore by $\left(P_{3}^{j}\right), \eta_{C}^{j} 1_{\Lambda \backslash C} \in V(n-1, \Lambda)$. Therefore we could extend an $(n-1)$-legal path from $1_{\Lambda}$ to $\eta_{C}^{j} 1_{\Lambda \backslash C}$ by adding the move from $\eta_{C}^{j} 1_{\Lambda \backslash C}$ to $\left(\eta_{C}^{j} 1_{\Lambda \backslash C}\right)^{z}$ and still have an $(n-1)$-legal path, which would imply $\left(\eta_{C}^{j} 1_{\Lambda \backslash C}\right)^{z} \in V(n-1, \Lambda)$, which is impossible.

We now deal with the case $z+X \subset D$.

We argue differently depending on the position of $z$.

- If $z$ is in the left part of $B$, we can use the fact that -1 is a stable direction. Indeed, $z+X$ would be at the right of $z$, hence $X$ would be contained in $\mathbb{N}^{*}$, which yields a contradiction.

- If $z$ is in the right part of $B$, we can use an argument similar to the one we used to deal with the case $z+X \subset C \cup \Lambda^{c}:\left(\eta_{D_{1}}^{j} 1_{D_{1}^{\prime} \backslash D_{1}}\right)^{z}$ would be in $V\left(n-1, D_{1}^{\prime}\right)$, which is impossible because it has a zero at $z$ and $z+\mathcal{P}_{n-1} \subset D_{1}^{\prime}$, so by $\mathcal{H}_{n-1}$ there is a contradiction. Indeed, $z+X$ would be contained in $D$ which is disjoint from $D_{1}^{\prime} \backslash D_{1}$, hence the move from $\eta_{D_{1}}^{j} 1_{D_{1}^{\prime} \backslash D_{1}}$ to $\left(\eta_{D_{1}}^{j} 1_{D_{1}^{\prime} \backslash D_{1}}\right)^{z}$ would be legal. Furthermore, $\left(\eta_{D_{1}}^{j} 1_{D_{1}^{\prime} \backslash D_{1}}\right)^{z}$ would coincide with $\eta^{j+1}$ on $D_{1} \cup B$, hence would contain at most $n-1$ zeroes, and by $\left(P_{4}^{j}\right) \eta_{D_{1}}^{j} 1_{D_{1}^{\prime} \backslash D_{1}} \in V\left(n-1, D_{1}^{\prime}\right)$. This would allow us to deduce $\left(\eta_{D_{1}}^{j} 1_{D_{1}^{\prime} \backslash D_{1}}\right)^{z} \in V\left(n-1, D_{1}^{\prime}\right)$, which is impossible.

We deduce that $z+X \subset D$ is impossible.

Consequently, $z \in B$ is impossible.

Case $z \in C$.

If $z \in C,\left(P_{1}^{j+1}\right)$ is true because $\eta_{\mathcal{P}_{n-1}}^{j+1}=\eta_{\mathcal{P}_{n-1}}^{j},\left(P_{2}^{j+1}\right)$ is true because $\eta_{B}^{j+1}=\eta_{B}^{j}$, and $\left(P_{4}^{j+1}\right)$ is true because $\eta_{D_{1}}^{j+1}=\eta_{D_{1}}^{j}$. The argument to prove $\left(P_{3}^{j+1}\right)$ is almost the same as the one that yielded $\left(\eta_{C}^{j} 1_{\Lambda \backslash C}\right)^{z} \in V(n-1, \Lambda)$ in the case $z \in B$ and $z+X \subset C \cup \Lambda^{c}$. We observe that as $z \in C$, we have $z+X \subset \Lambda^{c} \cup C \cup B$, and since $\left(P_{2}^{j}\right)$ implies $\eta_{B}^{j}=1_{B}$, we get $z+X \subset \Lambda^{c} \cup C$, so the move from $\eta_{C}^{j} 1_{\Lambda \backslash C}$ to $\eta_{C}^{j+1} 1_{\Lambda \backslash C}$ is legal. Furthermore, 
$\eta_{C}^{j+1} 1_{\Lambda \backslash C}$ contains at most $n-1$ zeroes, and by $\left(P_{3}^{j}\right)$ we have $\eta_{C}^{j} 1_{\Lambda \backslash C} \in V(n-1, \Lambda)$. This allows us to conclude that $\eta_{C}^{j+1} 1_{\Lambda \backslash C} \in V(n-1, \Lambda)$, which is $\left(P_{3}^{j+1}\right)$. Consequently, $\mathcal{H}_{j+1}^{\prime}$ holds.

Case $z \in D$.

If $z \in D,\left(P_{2}^{j+1}\right)$ is true because $\eta_{B}^{j+1}=\eta_{B}^{j}$, and $\left(P_{3}^{j+1}\right)$ is true because $\eta_{C}^{j+1}=\eta_{C}^{j}$.

Let us prove $\left(P_{1}^{j+1}\right)$.

If $z \in D_{1}$, then $\eta_{\mathcal{P}_{n-1}}^{j+1}=\eta_{\mathcal{P}_{n-1}}^{j}$, hence $\left(P_{1}^{j+1}\right)$ is true. We now suppose $z \in \mathcal{P}_{n-1}$. We prove $\left(P_{1}^{j+1}\right)$ using the fact that -1 is a stable direction. Indeed, it implies that $X$ is not contained in $\mathbb{N}^{*}$, hence since $X$ cannot contain 0 , it contains an element of $-\mathbb{N}^{*}$, thus there exists $z^{\prime} \in z+X$ with $z^{\prime}<z$. In addition, as $z \in \mathcal{P}_{n-1}$ we have $X \subset D \cup B$, and since by $\left(P_{2}^{j}\right) \eta_{B}^{j}=1_{B}$, we get $z+X \subset D$, therefore $z^{\prime} \in D$. Since $z^{\prime}<z, z^{\prime} \in \mathcal{P}_{n-1}$, and we have $\eta_{z^{\prime}}^{j+1}=\eta_{z^{\prime}}^{j}=0$. Consequently $\eta_{\mathcal{P}_{n-1}}^{j+1}$ contains a zero, hence $\left(P_{1}^{j+1}\right)$ is true.

Now let us prove $\left(P_{4}^{j+1}\right)$.

If $z \in \mathcal{P}_{n-1}$, then $\eta_{D_{1}}^{j+1}=\eta_{D_{1}}^{j}$, hence $\left(P_{4}^{j+1}\right)$ is true. In the case $z \in D_{1}$, we will prove $\left(P_{4}^{j+1}\right)$ with the arguments that gave $\left(\eta_{D_{1}}^{j} 1_{D_{1}^{\prime} \backslash D_{1}}\right)^{z} \in V\left(n-1, D_{1}^{\prime}\right)$ in the case $z \in B$ and $z+X \subset D$. Since $z \in D_{1}, z+X \subset D \cup B$, and as $\left(P_{2}^{j}\right)$ implies $\eta_{B}^{j}=1_{B}$ we get $z+X \subset D$, thus the move from $\eta_{D_{1}}^{j} 1_{D_{1}^{\prime} \backslash D_{1}}$ to $\eta_{D_{1}}^{j+1} 1_{D_{1}^{\prime} \backslash D_{1}}$ is legal, which allows to prove $\eta_{D_{1}}^{j+1} 1_{D_{1}^{\prime} \backslash D_{1}} \in V\left(n-1, D_{1}^{\prime}\right)$. Therefore $\left(P_{4}^{j+1}\right)$ is true.

This yields that $\mathcal{H}_{j+1}^{\prime}$ holds.

To conclude, $\mathcal{H}_{j+1}^{\prime}$ holds in all cases, which ends the proof of the lemma.

\section{The General CASE}

The reasoning to prove theorem 4 in general dimension is the same as in dimension 1 . However, the geometry is significantly more complicated, which will force us to introduce new notation.

Let $\mathcal{U}$ be a non supercritical unrooted update family. We will need the

Lemma 8. There exists $u_{1}, \ldots, u_{d} \in S^{d-1}$ stable directions for $\mathcal{U}$ and a normalized basis $\left\{v_{1}, \ldots, v_{d}\right\}$ of $\mathbb{R}^{d}$ such that for any $i \in\{1, \ldots, d\}, \mathbb{H}_{u_{i}}=\left\{\left(x_{1}, \ldots, x_{d}\right) \in \mathbb{R}^{d} \mid x_{i}>0\right\}$ in this basis.

To construct this basis, one takes $v_{i}$ orthogonal to all $u_{j}$ with $j \neq i$. A rigorous proof of the construction may be found in the appendix. From now on, we will use the coordinates of the basis $\left\{v_{1}, \ldots, v_{d}\right\}$, but when we say a site is in $\mathbb{Z}^{d}$, we will mean that its coordinates in the canonical basis are integers. For any $i \in\{1, \ldots, d\}$, since $u_{i}$ is a stable direction, there is no update rule contained in $\mathbb{H}_{u_{i}}$, hence no update rule such that all sites have a positive $i$-th coordinate.

We denote again by $r$ the range of the interactions: $r=\max \left\{\|x-y\|_{\infty} \mid x, y \in X \cup\{0\}, X \in \mathcal{U}\right\}$ (beware: the range is now defined in our new basis), and for all $n \in \mathbb{N}$, we set again $a_{n}=r\left(2^{n}-1\right)$ and $b_{n}=r n 2^{n-1}$. We now have to define $\mathcal{P}_{n}$ as follows (see figure 3):

$$
\mathcal{P}_{n}=\left\{s \in \mathbb{Z}^{d} \mid s=\left(s_{1}, \ldots, s_{d}\right), \forall i \in\{1, \ldots, d\},-a_{n} \leq s_{i} \leq b_{n}\right\} .
$$

We will again prove the theorem by induction: for all $n \in \mathbb{N}$, we denote

$$
\mathcal{H}_{n}=\text { "for any } \Lambda \subset \mathbb{Z}^{d} \text { such that } \mathcal{P}_{n} \subset \Lambda \text {, for any } \eta \in V(n, \Lambda), \eta_{0}=1 \text { ". }
$$

Proving $\mathcal{H}_{n}$ for all $n \in \mathbb{N}$ proves theorem 4. In order to do that, we need the following equivalent of lemma 7 ,

Lemma 9. Let $n \geq 1$ and suppose $\mathcal{H}_{n-1}$. Then, for all $\Lambda \subset \mathbb{Z}^{d}$ such that $\mathcal{P}_{n} \subset \Lambda$, for all $\eta \in V(n, \Lambda) \backslash\left\{1_{\Lambda}\right\}, \eta$ has at least one zero in $\Lambda \backslash \mathcal{P}_{n-1}$.

The proof of theorem 4 given lemma 9 is exactly the same as in the one-dimensional case, therefore it is enough to prove lemma 9 . 


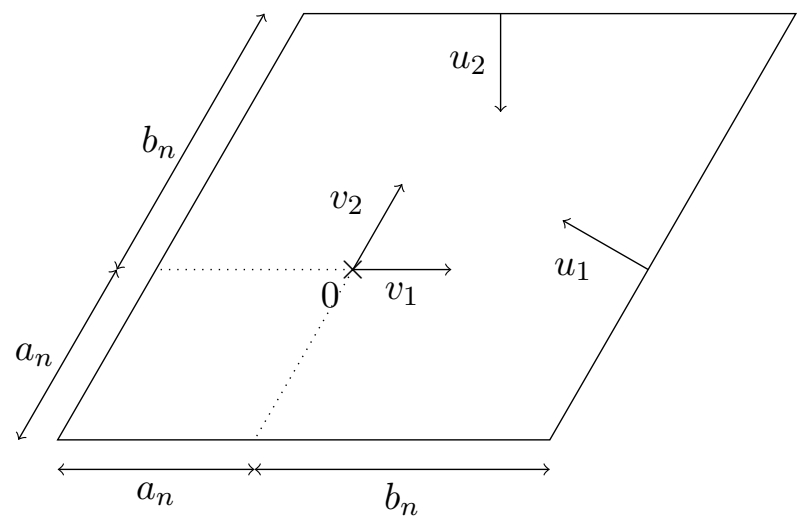

FiguRE 3. $\mathcal{P}_{n}$.

Proof of lemma 9. Let $n \geq 1$ and $\Lambda \subset \mathbb{Z}^{d}$ such that $\mathcal{P}_{n} \subset \Lambda$.

As in the one-dimensional case, we consider a configuration $\eta \in\{0,1\}^{\Lambda}$, different from $1_{\Lambda}$, containing at most $n$ zeroes, such that all of its zeroes are in $\mathcal{P}_{n-1}$, and we prove that $\eta \notin V(n, \Lambda)$. As previously, it is enough to let $\left(\eta^{j}\right)_{0 \leq j \leq m}$ be an $n$-legal path with $\eta^{0}=\eta$, and to show that $\eta^{m}$ cannot be $1_{\Lambda}$.

To this end, we denote for all $i \in\{1, \ldots, d\}$ (see figure 4):

$$
\begin{aligned}
D & =\left\{s \in \mathbb{Z}^{d} \mid s=\left(s_{1}, \ldots, s_{d}\right), \forall j \in\{1, \ldots, d\},-a_{n}+a_{n-1}+r \leq s_{j} \leq b_{n}-\left(b_{n-1}+r\right)\right\}, \\
B & =\left\{s \in \mathbb{Z}^{d} \mid s=\left(s_{1}, \ldots, s_{d}\right), \forall j \in\{1, \ldots, d\},-a_{n}+a_{n-1} \leq s_{j} \leq b_{n}-b_{n-1}\right\} \backslash D, \\
D_{i} & =\left\{s \in D \mid s=\left(s_{1}, \ldots, s_{d}\right), s_{i}>b_{n}-\left(b_{n-1}+a_{n-1}+r\right)\right\}, \\
D_{i}^{\prime} & =\left\{s \in \mathcal{P}_{n} \mid s=\left(s_{1}, \ldots, s_{d}\right), s_{i}>b_{n}-\left(b_{n-1}+a_{n-1}+r\right)\right\}
\end{aligned}
$$

and $C=\Lambda \backslash(B \cup D)$. We also notice that as in dimension $1,-a_{n}+a_{n-1}+r=-a_{n-1}$ and $b_{n}-\left(b_{n-1}+a_{n-1}+r\right)=b_{n-1}$, hence

$$
\mathcal{P}_{n-1}=\left\{s \in \mathbb{Z}^{d} \mid s=\left(s_{1}, \ldots, s_{d}\right), \forall j \in\{1, \ldots, d\},-a_{n}+a_{n-1}+r \leq s_{j} \leq b_{n}-\left(b_{n-1}+a_{n-1}+r\right)\right\}
$$

thus $\mathcal{P}_{n-1}=D \backslash\left(\bigcup_{i=1}^{d} D_{i}\right)$.

As in the one-dimensional case, $B$ will be a buffer zone preventing the zeroes of $C$ and $D$ from interacting. In that case, the main reason for which no zero could appear in $B$ was that a zero remained trapped in $\mathcal{P}_{n-1}$, hence there were at most $n-1$ zeroes elsewhere, and $\mathcal{H}_{n-1}$ limited their possible positions.

Here we cannot keep a zero in $\mathcal{P}_{n-1}$, but we can keep a zero in all the $D \backslash D_{i}$. Indeed, initially there is at least a zero in $\mathcal{P}_{n-1} \subset D \backslash D_{i}$, and at any time, a zero of $D \backslash D_{i}$ with the lowest $i$-th coordinate among the zeroes of $D \backslash D_{i}$ will need an update rule full of zeroes in order to disappear, hence a zero with a $i$-th coordinate as low as its own because there is no update rule whose sites all have positive $i$-th coordinate (this is the reason for which we work in the basis $\left.\left\{v_{1}, \ldots, v_{d}\right\}\right)$. This zero cannot be in $B$ since $B$ remains full of ones, hence it is in $D \backslash D_{i}$ and so remains in $D \backslash D_{i}$ at the next step of the path.

This will have the same practical consequences as the zero trapped in $\mathcal{P}_{n-1}$ had in the one-dimensional case: the presence of a zero in each of the $D \backslash D_{i}$ prevents $\eta^{m}$ from being $1_{\Lambda}$; the $n-1$ zeroes that any of the $D_{i}$, or $C$, may contain will not escape the $D_{i}$ or $C$. Moreover, for any $i \in\{1, \ldots, d\}$, the argument that in dimension 1 prevented the zeroes of $\mathcal{P}_{n-1}$ from escaping to the left part of $B$ because there were no update rule contained in $\mathbb{N}^{*}$ will here prevent zeroes from escaping $D$ via the face with the lowest $i$-th coordinate to enter $B$, since there is no update rule whose sites all have positive $i$-th coordinates. Therefore the buffer zone $B$ will be preserved. 


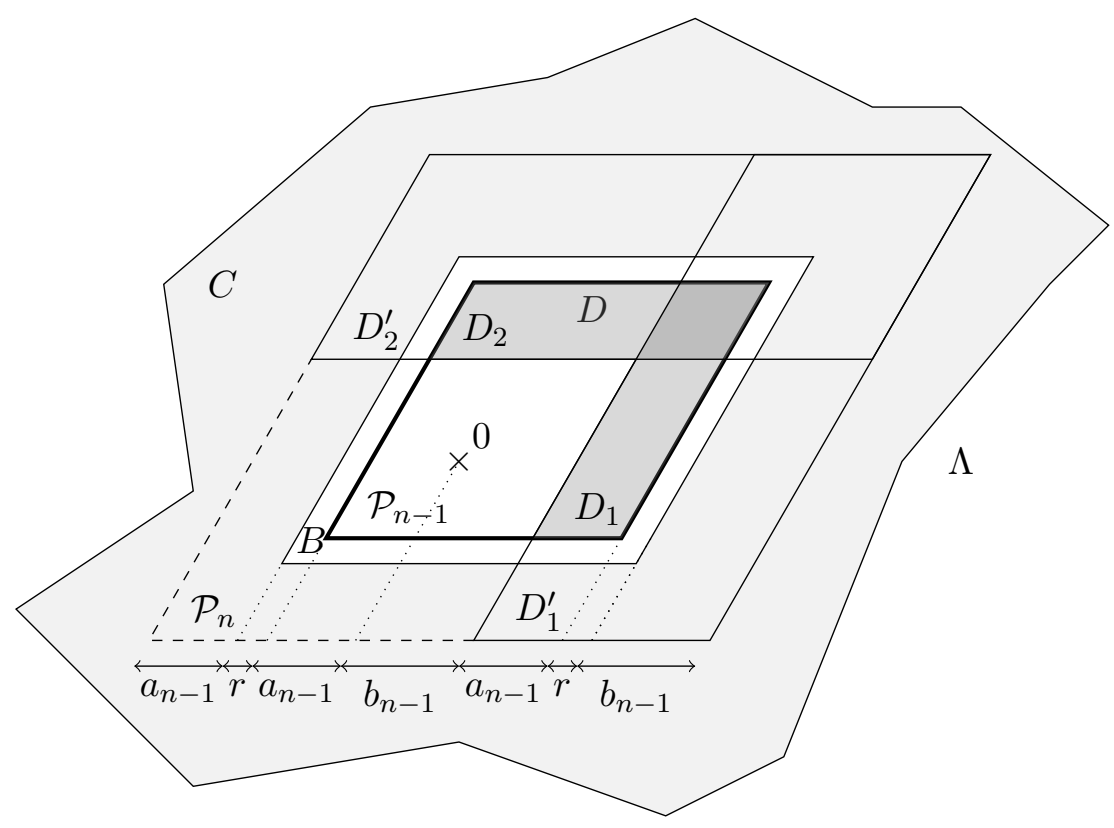

Figure 4. The setting of lemma $9 . C$ is in light gray, $D_{1}$ and $D_{2}$ are in darker gray, $D$ is the region with the thick outline.

The details of the proof are very similar to those of the proof of lemma 7 , therefore we only detail the changes. We have to change the induction hypothesis $\mathcal{H}_{j}^{\prime}$, which becomes:

$\left(P_{1}^{j}\right)$ For all $i \in\{1, \ldots, d\}, \eta_{D \backslash D_{i}}^{j}$ contains a zero.

$\left(P_{2}^{j}\right) \eta_{B}^{j}=1_{B}$.

$\left(P_{3}^{j}\right) \eta_{C}^{j} 1_{\Lambda \backslash C} \in V(n-1, \Lambda)$.

$\left(P_{4}^{j}\right)$ For all $i \in\{1, \ldots, d\}, \eta_{D_{i}}^{j} 1_{D_{i}^{\prime} \backslash D_{i}} \in V\left(n-1, D_{i}^{\prime}\right)$.

When proving the induction, the more complicated geometry forces us to refine the proof of the fact that the case $z \in B$ and $z+X \subset D$ is impossible. Since $z \in B$, if we denote by $\left(z_{1}, \ldots, z_{d}\right)$ the coordinates of $z$, there would exist $i \in\{1, \ldots, d\}$ such that $z_{i}<-a_{n}+a_{n-1}+r$ ( $z$ is "at the left of $B$ for the $i$-th coordinate") or $z_{i}>b_{n}-\left(b_{n-1}+r\right)(z$ is "at the right of $B$ for the $i$-th coordinate").

- If $z_{i}<-a_{n}+a_{n-1}+r$, we notice that $z+X \subset D$ would imply that $X \subset\left\{\left(x_{1}, \ldots, x_{d}\right) \in \mathbb{R}^{d} \mid x_{i}>0\right\}$, which is impossible because there is no update rule whose sites all have a positive $i$-th coordinate.

- If $z_{i}>b_{n}-\left(b_{n-1}+r\right)$, we can use the same argument as in dimension 1 with $D_{i}$ replacing $D_{1}$, which yields a contradiction.

We deduce a contradiction in both cases, therefore $z+X \subset D$ is indeed impossible.

Finally, the proof of $\left(P_{1}^{j+1}\right)$ when $z \in D$ also deserves a refinement. We set $i \in\{1, \ldots, d\}$, let us prove that $\eta_{D \backslash D_{i}}^{j+1}$ contains a zero. If $z \in D_{i}$, then $\eta_{D \backslash D_{i}}^{j+1}=\eta_{D \backslash D_{i}}^{j}$, hence by $\left(P_{1}^{j}\right) \eta_{D \backslash D_{i}}^{j+1}$ contains a zero. If $z \in D \backslash D_{i}$, we use the fact that $X$ cannot be contained in $\left\{\left(x_{1}, \ldots, x_{d}\right) \in \mathbb{R}^{d} \mid x_{i}>0\right\}$, hence there exists a site $z^{\prime} \in z+X$ such that the $i$-th coordinate of $z^{\prime}$ is less than or equal to the $i$-th coordinate of $z$. Moreover, we observe that $z+X \subset D \cup B$, and by $\left(P_{2}^{j}\right) \eta_{B}^{j}=1_{B}$, thus $z+X \subset D$, so $z^{\prime} \in D$. Since the $i$-th coordinate of $z^{\prime}$ is less than or equal to the $i$-th 


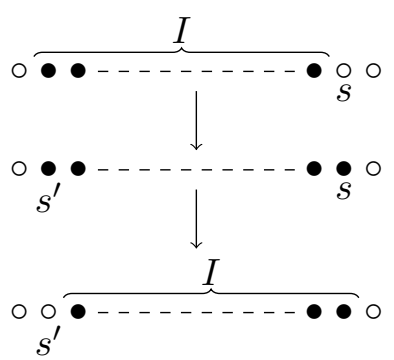

Figure 5. A move towards the right of an interval $I$ of zeroes for a one-dimensional supercritical unrooted update family. Zeroes are represented by $\bullet$ and ones by 0 .

coordinate of $z$ and $z \in D \backslash D_{i}, z^{\prime} \in D \backslash D_{i}$. Furthermore, we have $\eta_{z^{\prime}}^{j+1}=\eta_{z^{\prime}}^{j}=0$. Consequently, $\eta_{D \backslash D_{i}}^{j+1}$ contains a zero. Therefore $\eta_{D \backslash D_{i}}^{j+1}$ contains a zero for all $i \in\{1, \ldots, d\}$, hence $\left(P_{1}^{j+1}\right)$ is true.

\section{Sketch of the proof of proposition 6}

For $d=1$, if $\mathcal{U}$ is a supercritical unrooted family, it has no stable direction, therefore there must be an update rule contained in $\mathbb{N}^{*}$ and another contained in $-\mathbb{N}^{*}$. Consequently, as illustrated by figure 5 , if we have an interval $I \subset \mathbb{Z}$ of zeroes that is sufficiently large, the site $s$ at the right of $I$ can be put at zero with a legal move. Then the site $s^{\prime}$ at the left of the interval can be put at one by a legal move, and $I$ has moved to the right by one unit. By having $I$ starting from outside the domain (where there are only zeroes) and moving towards the origin in that way, one can put the origin at zero using a bounded number of zeroes, whatever the size of the domain.

For $d=2$ the mechanism is similar, but requires a more complex construction. In section 5 of [5] (see in particular figure 5 and lemma 5.5 therein), it is proven that if $\mathcal{U}$ is an update family with a semicircle of unstable directions centered on direction $u$, it is possible to construct a "droplet": a finite set of zeroes that even if all other sites are at 1 , allows us to put more sites at zero in direction $u$ with legal moves, creating a bigger droplet of the same shape, as illustrated on part (a) of figure 6. It is the shape of the part of the droplet towards direction $u$ that enables its growth towards this direction. If $\mathcal{U}$ is supercritical unrooted, its stable directions are contained in a hyperplane of $\mathbb{R}^{2}$, which means a straight line, hence there are at most two stable directions, and they must then be opposite. Therefore, there exists two opposite semicircles containing no stable direction, with middles $u$ and $-u$. We can use the construction of $[5]$ to build two droplets, corresponding to the two semicircles, that can grow respectively in the directions $u$ and $-u$ (see part (b) of figure 6). Using these two droplets, we can get a combined droplet that can grow in both directions $u$ and $-u$ (part (c) of figure 6). Moreover, since our rules allow any change of site state to be reversed, the droplet will also be able to shrink in these directions. Therefore, by having the droplet grow in direction $u$ and shrink in direction $-u$, we can make it move towards direction $u$ (see part (d) of figure (6). This allows us to bring it to the origin using a bounded number of zeroes as we did with the interval we had for $d=1$.

For $d \geq 3$, we expect a similar phenomenon to occur, but we cannot prove it because an equivalent of the construction of [5] is not available yet.

\section{APPENDIX: PROOF OF LEMMA 8}

By assumption, the update family $\mathcal{U}$ is not supercritical unrooted, hence its stable directions are not contained in any hyperplane of $\mathbb{R}^{d}$. Therefore, there exists stable directions $u_{1}, \ldots, u_{d}$ of $\mathcal{U}$ that form a basis of $\mathbb{R}^{d}$. For any 


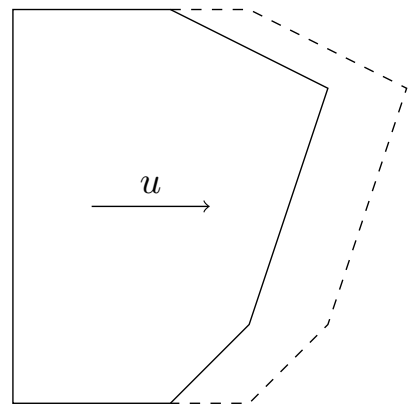

(a)

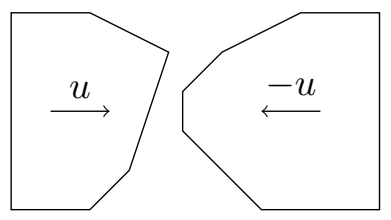

(b)

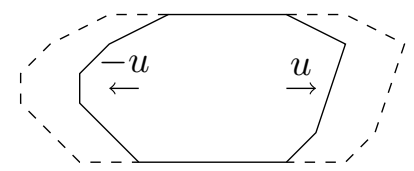

(c)

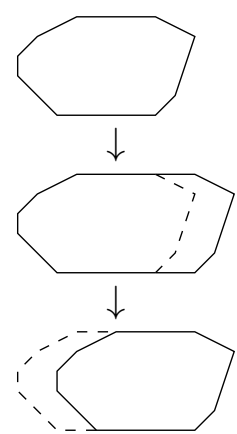

(d)

Figure 6. The construction of a droplet of zeroes for a two-dimensional supercritical unrooted update family that can move towards $u$ and $-u$. (a) The shape delimited by the solid line is the droplet of [5], that can grow to the shape delimited by the dashed line. (b) The droplets corresponding to the semicircles centered at $u$ and $-u$. (c) The combined droplet. (d) A move of the combined droplet to the right.

$u \in S^{d-1}$, we denote $\mathcal{H}_{u}$ the hyperplane orthogonal to $u: \mathcal{H}_{u}=\left\{x \in \mathbb{R}^{d} \mid\langle x, u\rangle=0\right\}$. Then, for any $i \in\{1, \ldots, d\}$, $\bigcap_{j \neq i} \mathcal{H}_{u_{j}}$ is a straight line 11 For any $i \in\{1, \ldots, d\}$, we define $v_{i}$ as a unitary vector in $\bigcap_{j \neq i} \mathcal{H}_{u_{j}}$.

We are going to show that $\left\{v_{1}, \ldots, v_{d}\right\}$ is a basis of $\mathbb{R}^{d}$. For any set of vectors $\left\{w_{1}, \ldots, w_{m}\right\} \subset \mathbb{R}^{d}$, we denote $\operatorname{Vect}\left\{w_{1}, \ldots, w_{m}\right\}$ the vector space generated by $\left\{w_{1}, \ldots, w_{m}\right\}$. It is enough to prove that Vect $\left\{v_{1}, \ldots, v_{d}\right\}=\mathbb{R}^{d}$. In order to do that, we take $v \in \mathbb{R}^{d}$ a vector orthogonal to $\operatorname{Vect}\left\{v_{1}, \ldots, v_{d}\right\}$. We are going to show that $v$ must be the null vector. For all $i \in\{1, \ldots, d\}, v$ is orthogonal to $v_{i}$. Moreover, the vector space orthogonal to $v_{i}$ has dimension $d-1$. Furthermore, $v_{i} \in \bigcap_{j \neq i} \mathcal{H}_{u_{j}}$, hence the $u_{j}, j \neq i$ are orthogonal to $v_{i}$. Hence, as the $u_{j}, j \neq i$ are $d-1$ linearly independent vectors, the vector space orthogonal to $v_{i}$ is $\operatorname{Vect}\left\{u_{1}, \ldots, u_{i-1}, u_{i+1}, \ldots, u_{d}\right\}$. This implies that $v$ belongs to $\operatorname{Vect}\left\{u_{1}, \ldots, u_{i-1}, u_{i+1}, \ldots, u_{d}\right\}$, for any $i \in\{1, \ldots, d\}$. As $\left\{u_{1}, \ldots, u_{d}\right\}$ is a basis of $\mathbb{R}^{d}$, this yields $v=0$. Consequently, the vector space orthogonal to $\operatorname{Vect}\left\{v_{1}, \ldots, v_{d}\right\}$ is reduced to $\{0\}$. We deduce $\operatorname{Vect}\left\{v_{1}, \ldots, v_{d}\right\}=\mathbb{R}^{d}$, thus $\left\{v_{1}, \ldots, v_{d}\right\}$ is a basis of $\mathbb{R}^{d}$.

We want a basis such that for any $i \in\{1, \ldots, d\}, \mathbb{H}_{u_{i}}=\left\{\left(x_{1}, \ldots, x_{d}\right) \in \mathbb{R}^{d} \mid x_{i}>0\right\}$. In $\left\{v_{1}, \ldots, v_{d}\right\}$, $\left\{\left(x_{1}, \ldots, x_{d}\right) \in \mathbb{R}^{d} \mid x_{i}=0\right\}$ is generated by the vectors $v_{1}, \ldots, v_{i-1}, v_{i+1}, \ldots, v_{d}$, which are $d-1$ linearly independent vectors belonging to the hyperplane $\mathcal{H}_{u_{i}}$ of $\mathbb{R}^{d}$, hence they generate $\mathcal{H}_{u_{i}}$. This implies $\mathcal{H}_{u_{i}}=\left\{\left(x_{1}, \ldots, x_{d}\right) \in\right.$ $\left.\mathbb{R}^{d} \mid x_{i}=0\right\}$. Therefore, $\mathbb{H}_{u_{i}}$ is either $\left\{\left(x_{1}, \ldots, x_{d}\right) \in \mathbb{R}^{d} \mid x_{i}>0\right\}$ or $\left\{\left(x_{1}, \ldots, x_{d}\right) \in \mathbb{R}^{d} \mid x_{i}<0\right\}$. If $\mathbb{H}_{u_{i}}=$ $\left\{\left(x_{1}, \ldots, x_{d}\right) \in \mathbb{R}^{d} \mid x_{i}<0\right\}$, we replace $v_{i}$ with $-v_{i}$. Thus we get $\mathbb{H}_{u_{i}}=\left\{\left(x_{1}, \ldots, x_{d}\right) \in \mathbb{R}^{d} \mid x_{i}>0\right\}$.

This method allows us to obtain a basis $\left\{v_{1}, \ldots, v_{d}\right\}$ satisfying that for any $i \in\{1, \ldots, d\},\left\|v_{i}\right\|_{2}=1$ and $\mathbb{H}_{u_{i}}=\left\{\left(x_{1}, \ldots, x_{d}\right) \in \mathbb{R}^{d} \mid x_{i}>0\right\}$.

\section{ACKNOWLEDGMENTS}

I would like to thank my PhD advisor Cristina Toninelli for introducing me to the subject and helping me along this work. I also would like to thank Robert Morris for suggesting that the result could extend beyond dimension 2, as well as Fabio Martinelli and Ivailo Hartarsky for their helpful suggestions about the presentation of this work.

\footnotetext{
${ }^{1}$ Indeed, $\bigcap_{j \neq i} \mathcal{H}_{u_{j}}$ is the intersection of $d-1$ hyperplanes in $\mathbb{R}^{d}$, hence it contains a straight line. Furthermore, $\bigcap_{j \neq i} \mathcal{H}_{u_{j}}$ is orthogonal to the $u_{j}, j \neq i$, and since $\left\{u_{1}, \ldots, u_{d}\right\}$ is a basis of $\mathbb{R}^{d},\left\{u_{j}: j \neq i\right\}$ generate a vector space of dimension $d-1$. Therefore $\bigcap_{j \neq i} \mathcal{H}_{u_{j}}$ is orthogonal to a vector space of dimension $d-1$. Consequently, it is at most a straight line.
} 


\section{REFERENCES}

[1] David Aldous and Persi Diaconis. The asymmetric one-dimensional constrained Ising model: rigorous results. Journal of statistical physics, 107(5-6):945-975, June 2002.

[2] Paul Balister, Béla Bollobás, Michał Przykucki, and Paul Smith. Subcritical $\mathcal{U}$-bootstrap percolation models have non-trivial phase transitions. Transactions of the American Mathematical Society, 386:7385-7411, January 2016.

[3] Ludovic Berthier and Giulio Biroli. Theoretical perspective on the glass transition and amorphous materials. Reviews of modern physics, 83:587-645, April-June 2011.

[4] Béla Bollobás, Hugo Duminil-Copin, Robert Morris, and Paul Smith. Universality of two-dimensional critical cellular automata. Proceedings of the London Mathematical Society, to appear.

[5] Béla Bollobás, Paul Smith, and Andrew Uzzell. Monotone cellular automata in a random environment. Combinatorics, probability and computing, 24(4):687-722, July 2015.

[6] Nicoletta Cancrini, Fabio Martinelli, Cyril Roberto, and Cristina Toninelli. Kinetically constrained spin models. Probability theory and related fields, 140(3-4):459-504, 2008.

[7] Nicoletta Cancrini, Fabio Martinelli, Roberto H. Schonmann, and Cristina Toninelli. Facilitated oriented spin models : some non equilibrium results. Journal of statistical physics, 138(6):1109-1123, March 2010.

[8] Fan Chung, Persi Diaconis, and Ronald Graham. Combinatorics for the East model. Advances in applied mathematics, 27(1):192206, July 2001

[9] J. Jäckle and S. Eisinger. A hierarchically constrained kinetic ising model. Zeitschrift für Physik B Condensed Matter, (84):115-124, 1991.

[10] Laure Marêché, Fabio Martinelli, and Cristina Toninelli. Exact asymptotics for Duarte and supercritical rooted kinetically constrained models. arXiv:180\%.07519, 2018.

[11] Fabio Martinelli, Robert Morris, and Cristina Toninelli. Universality results for kinetically constrained spin models in two dimensions. Communications in mathematical physics, to appear.

[12] Fabio Martinelli and Cristina Toninelli. Towards a universality picture for the relaxation to equilibrium of kinetically constrained models. Annals of probability, to appear.

[13] Robert Morris. Bootstrap percolation, and other automata. European journal of combinatorics, 66:250-263, December 2017.

[14] Felix Ritort and Peter Sollich. Glassy dynamics of kinetically constrained models. Advances in physics, 52(4):219-342, February 2003.

[15] Peter Sollich and Martin Evans. Glassy time-scale divergence and anomalous coarsening in a kinetically constrained spin chain. Physical review letters, 83:3238, 1999.

Laure Marêché, LPSM umR 8001, Université Paris Diderot, Sorbonne Paris Cité, CNRS, 75013 Paris, France E-mail address: mareche@lpsm.paris 